\title{
SATISFACCIÓN FAMILIAR EN MUJERES JÓVENES
}

\section{Family satisfaction in young women}

\author{
Jenny Quezada Zevallos*, Elsa Zavala Bustamante**, \\ Martha Lenti Canepa***
}

\begin{abstract}
Resumen
El concepto de satisfacción familiar se halla poco analizado en sus dimensiones, el objetivo del presente estudio es realizar un análisis de las dimensiones contenidas en la Escala de Satisfacción familiar por Adjetivos (ESFA). Estudio de tipo descriptivo, la muestra estuvo conformada por 85 mujeres de edades 16 a 24 años., el instrumento el ESFA.

Resultados: La escala tiene una validez de contenido adecuada, validado a través de la técnica de criterio de jueces. La confiabilidad estadística global es alta (Alpha de Cronbach .917 y un Coeficiente Guttman Split-Half de .934).

Los ítems de la escala ESFA, de acuerdo a un jurado conformado por 4 especialistas en el área de familia, determinaron agrupar los mismos en 2 dimensiones cada una de ellas a su vez. conformada por dos elementos: I. Amor Familiar (Holding y Sentido de pertenencia a la familia) y II. Felicidad (Alegría y Tranquilidad). El grupo de estudio presenta una Satisfacción familiar a nivel Alto, y en todas las dimensiones y elementos un nivel Alto.
\end{abstract}

Palabras clave: Satisfacción familiar, amor familiar, holding, sentido de pertenencia a la familia, felicidad, alegría, tranquilidad, mujeres.

\begin{abstract}
The concept of family satisfaction is rarely analyzed in its dimensions; the objective of this study is to analyze the dimensions contained in the Family Satisfaction Scale by Adjectives, known by its acronym in Spanish - ESFA. This is a descriptive study; the sample consisted of 85 women between 16 to 24 years old. The instrument to be used is the ESFA.

Results: The scale has an adequate content validity, validated through the technique of judging criteria. The global statistical reliability is high (Alpha de Cronbach 917 y un Coeficiente Guttman Split-Half de .934).

The ESFA scale items, according to a jury of 4 specialists in the Family area. They grouped the same ones in 2 dimensions each of them conformed by two elements: I. Family Love (Holding and Sense of belonging to the family) and II. Happiness (Joy and Tranquility). The group of study presents a High level of Family Satisfaction, and in all dimensions and elements a High level.
\end{abstract}

Keywords: Family satisfaction, family love, holding, sense of belonging to the family, happiness, joy, tranquility, women.

\footnotetext{
* Psicóloga. Universidad Femenina del Sagrado Corazón. jennyquezadaz@unife.edu.pe

** Psicóloga. Universidad Femenina del Sagrado Corazón.milagroszb@gmail.com

*** Psicóloga. Universidad Femenina del Sagrado Corazón. psico.marthalenti@gmail.com
} 


\section{INTRODUCCIÓN \\ CONCEPTUALIZACIÓN FAMILIAR}

En los últimos 25 años, innumerables investigaciones han dado evidencia de la influencia que tiene el contexto familiar en el desarrollo psicológico de la persona.

"La familia, es la institución más antigua y resistente de todos los grupos humanos, aunque ha ido variando tanto en sus funciones como en su estructura a lo largo de la historia. Cumple entre otras, las funciones de crianza, educación y cuidado. Asimismo, satisface las necesidades básicas como alimento, vivienda y dedica parte de su tiempo a la atención de los más necesitados, como niños, ancianos, personas con discapacidad y enfermos. Es el refugio que brinda afecto, soporte emocional, económico y protección" (Bermúdez C, Brik E, p19 Terapia Familiar Sistémica).

La denominación de familia generalmente está referida a un grupo de personas que se encuentran vinculadas por lazos de consanguinidad, el cual es el sistema más antiguo, del que tenemos referencia; pero también está la vinculación de tipo afectiva, económica, social; dando de éste modo diversos tipos de grupos denominados: familia.

Arranz (2004), propone que existen variables de carácter contextual o interactivo que influyen significativamente. Las variables contextuales son las que no reflejan interacciones sociales directas; pero que influyen sobre ellas, como por ejemplo el nivel educativo de los padres. Las variables interactivas, se conjugan con las relaciones sociales directas, como los estilos de crianza de los padres; éstas se ubican dentro del sistema familiar, ya sea en uno de los subsistemas o entre dos subsistemas.

Por otro lado, dentro de las funciones de la familia, se puede destacar fundamentalmente dos: el sentido de contener a los miembros que la conforman, en diversos aspectos, tales como: afecto, cultura, transmisión de valores y respaldo material; sin embargo, cabe mencionar que para que se produzca la función de respaldo y cuidado a sus miembros; el grupo debe sentirse identificado y perteneciente al mismo; se tiene así la segunda función que cumple la familia.
Bermúdez, C y Brik E (2010) señalan que en el ámbito de las relaciones familiares, se requiere considerar la funcionalidad del sistema en relación a dos aspectos relevantes: la de sostenimiento y la de pertenencia. De igual modo, los referidos autores señalan que la funcionalidad de una familia, también se define por la consecución de los pasos requeridos $\mathrm{y}$ necesarios que generen individuos debidamente integrados a una estructura social, con adecuada posibilidad de adaptarse a los cambios que las diversas etapas del ciclo vital así les exija; haciéndose conscientes de la importancia de desarrollar una comunicación suficientemente fluida, que se base no sólo en el respecto sino también que pueda dar cabida al emocionar de sus integrantes de modo adecuado.

Icart y Freixas (2013) sostienen citando a J. Thomas (1995); sobre el sentido contenedor de la familia, que es una estructura donde reconoce al sistema parental como el artífice principal de llevar a cabo la función de sostenimiento grupal y a su vez individual de quienes conforman el sistema. De cómo se lleve a cabo dicha función, se alimentará el sentido de satisfacción psíquica que cada uno de sus miembros comporte.

Sánchez Quiroga (1995) afirman que el grado de satisfacción de los miembros se considera un predictor del funcionamiento familiar; considerando que éste resulta del juego de interacciones que se dan al interior de las familias, relacionando la ausencia de conflictos con un mayor grado de satisfacción familiar.

La familia a lo largo de su ciclo vital, va planteando sus propios modelos de relación, estrategias de cambio y normas, de igual modo, van desarrollando sus propios recursos aportados por todos sus miembros que van asimilando del mismo entorno en el que la familia se desenvuelve (sociedad) o de su propia historia familiar. Durante este proceso de desarrollo, la familia, van planteando sus propias metas, que en un inicio pueden ser asignadas socialmente y luego las van interiorizando para formar su propia organización, retroalimentándose de las expectativas del logro de sus propias metas, de la eficacia y éxito de las estrategias utilizadas que son muy únicos y que definen su identidad como familia y además contribuyen significativamente al sentido de individuación, de los miembros que forman parte de 
ella. Este proceso en el que se van definiendo las pautas de relación y transacciones propias de la familia, puede traer consigo bienestar y satisfacción con su vida o contrariamente, infelicidad o inadaptación.

En tal sentido, La Fuente Benaches, María Josefa, refiere;

"La familia es como un banco de pruebas, y sólo si dentro de ella el niño encuentre la ayuda necesaria para adquirir una cierta soltura y desarrolla confianza en sus propias capacidades, podrá aventurarse a explorar y conocer el exterior con cierta garantía de éxito..." (La Fuente Benaches, pág. 37)

Cuando las familias no llevan a cabo los procesos de adaptación y transformación que cada etapa del ciclo vital le demanda; suele atascarse en alguna parte de éste, produciéndose una crisis y muchas veces manifestándose la misma en la aparición de un "síntoma" concretado en alguno de sus miembros, que de algún modo da la voz de alarma, de que el sistema referencial, requiere de una atención o revisión interna del funcionamiento familiar.

Respecto a la relevancia del estudio de satisfacción familiar, Guillen, R. (2011) menciona la importancia de comprender aquellos sentimientos y actitudes que se han desarrollado acerca de la propia familia y que se hace evidente en los diferentes tipos de familias. El contar con una medida del funcionamiento familiar puede reflejar el bienestar psicológico de sus miembros como contraparte de los indicadores psicopatológicos que se podrían presentar.

En diferentes investigaciones realizadas con familias, se enfatiza la importancia del rol que cumplen los padres en el desarrollo emocional y conductual de sus hijos. Salazar Alvarez, Y. (2013) hace referencia a Steinberg (2001), quien destaca que en la adolescencia temprana pueden aparecer conflictos entre padres e hijos; sin embargo pese a ello se siguen manteniendo relaciones estrechas y afectuosas.

Salazar Alvarez Y., Veytia López M., Marquez Mendoza O. y Huitrón Bravo G. (2013) publican una investigación Relación entre satisfacción con el ambiente familiar y depresión en adolescentes, en una muestra de 448 estudiantes de bachillerato entre 14 y 19 años, de una institución pública del Estado de México. Para obtener información respecto a las variables de estudio, se aplicó el Cuestionario para el Diagnóstico de Cuadros depresivos (CDC) y la Escala de Satisfacción Familiar por Adjetivos (ESFA). Los resultados muestran que los adolescentes con insatisfacción familiar tuvieron mayores prevalencias de ansiedad (15.3\%) y depresión (35.1\%) comparándolos con los adolescentes con satisfacción familiar. Al correlacionar las variables satisfacción familiar y depresión, se encontró una relación negativa significativa, que indica que mientras mayor es la insatisfacción familiar, es mayor la probabilidad que el adolescente sufra de depresión.

Céspedes Lorena, Roveglia Antonella y Coppari Norma; (2014) realizaron una investigación Satisfacción familiar en adolescentes de un colegio privado de Asunción, en la que evalúan y describen la satisfacción familiar en una muestra de 140 estudiantes hombre y mujeres entre 12 y 18 años de un Colegio Privado de la ciudad de Asunción. Los resultados obtenidos en la Escala de Satisfacción Familiar por Adjetivos, indican que el $30.7 \%$ de estudiantes muestran un nivel bajo de satisfacción familiar, el $28.6 \%$ el nivel alto y el $40.7 \%$ alcanzan el nivel promedio de satisfacción familiar; concluyendo que probablemente exista mayor número de familia con un clima familiar en el que predomina el enojo, la frustración, la desilusión, tristeza y depresión.

La satisfacción familiar, tema de nuestro interés, está relacionada a los sentimientos despertados a partir de las interacciones (verbales y/o físicas) que ocurren entre el sujeto y los otros miembros de la familia (Barraca y López-Yarto, 2010).

El grado de satisfacción familiar de los miembros puede ser un predictor del funcionamiento familiar, considerando que éste resulta del juego de interacciones que se dan en la familia, si éste último presenta buenos niveles, habría ausencia de conflicto y una satisfacción familiar mayor (Sanchez y Quiroga, 1995).

En tal sentido, una mayor satisfacción con la vida familiar, estaría relacionada con mayor frecuencia e 
intensidad de experiencias afectivas de amor, cariño, afecto, alegría y felicidad; mientras que una menor satisfacción con la vida de familia se relacionaría con mayores experiencias de coraje, enojo, frustración, desilusión, tristeza y depresión (Luna, 2011).

Siguiendo la línea de pensamiento de la Psicología Positiva, las experiencias afectivas positivas que se viven al interior de la familia, dependen del individuo, de la forma cómo potencia las fuerzas positivas internas, sin perder de vista las influencias de la sociedad y la cultura en la cual está inmerso. La fuerza sociocultural va modelando el comportamiento humano, y propicia que se establezcan ciertos comportamientos que se generalizan y se expresan a través de los valores, actitudes, creencias y variables de la personalidad.

Uno de los temas que aborda es la Felicidad, término que se relaciona directamente con el bienestar subjetivo, el bienestar psicológico y satisfacción con la vida.

Alarcón Reynaldo (2008), define el término Felicidad; como un estado afectivo de satisfacción plena que experimenta subjetivamente un individuo en posesión de un bien deseado.

La felicidad es un sentimiento de satisfacción que vive una persona en su mundo interno y subjetivo que lo hace único; es temporal; supone la posesión de un bien deseado y este bien puede ser material, ético, estético, psicológico; etc. este bien tienen un valor intrínseco en la persona; pero no se descarta que en algún momento y en una determinada cultura, se establezcan aspiraciones colectivas respecto al deseo de algún bien.

Ser feliz en el contexto familiar, significa establecer vínculos positivos y nutritivos con los padres, hermanos y personas que conviven en el entorno inmediato. Un adecuado vínculo permite a la persona desarrollar la capacidad empática, y favorecer el aprecio de parte de los demás, todo en un contexto social que propicia la posibilidad de cumplir con ciertas tareas que repercuten en el beneficio de los demás.

El objetivo del estudio que a continuación se presenta, contempla las siguientes dimensiones: Amor
Familiar y Felicidad, que a su vez se desagregan en: Holding y Sentido de Pertenencia a la Familia, por un lado y Alegría y Tranquilidad y confianza por otro. Estos elementos han sido conceptualizados dentro de la Escala de Satisfacción Familiar (ESFA).

A continuación se detalla cada una de las dimensiones mencionadas con sus respectivos elementos.

\section{DEFINICIONES OPERACIONALIZACIÓN DE TERMINOS IMPORTANTES:}

\section{- AMOR FAMILIAR:}

Sentimiento surgido entre los miembros de un sistema familiar, que los ayuda a conectarse, relacionarse yenlazarse, otorgandoreconocimiento al existir del "otro" en sus propias vidas.

- SENTIDO DE PERTENENCIA A LA FAMILIA: Conjunto de acciones, valoraciones, sentimientos y reconocimientos que experimentan los individuos que conforman un sistema familiar y del cual se consideran parte activa.

- SOSTENIMIENTO FAMILIAR / HOLDING: Proceso que realiza el sistema adulto, generalmente los padres, en función de sus hijos u otros miembros de la familia que así lo requiriesen, con la finalidad de brindar el necesario apoyo en favor del desarrollo integral de sus miembros.

\section{- SATISFACCIÓN FAMILIAR:}

Se entiende como un conjunto de sentimientos que se evidencian en las personas al estar con su familia, que son el resultado de una serie de interacciones positivas, nutritivas y gratificantes que se establecen y se mantienen al interior de ella. Estas relaciones son vividas de manera propia y única en cada miembro de la familia.

\section{- FELICIDAD:}

Es el estado afectivo de satisfacción y regocijo que experimenta subjetivamente el individuo al alcanzar sus expectativas y logros o por el bienestar que vive al sentirse inmerso en un medio que lo retroalimenta de manera positiva y afectiva. 


\section{- CONFIANZA}

Se refiere a la creencia y seguridad que una persona desarrolla respecto de sí misma, a una situación o a la forma de actuar de otra persona. Ésta puede generarse de una manera consciente a partir de situaciones particulares y/o experiencias vividas en cada individuo. Es una vivencia única e individual que se relaciona con un sentimiento de tranquilidad focalizado en las expectativas de una acción futura y que puede reforzarse o debilitarse en función de las acciones de las otras personas o de las circunstancias que enfrenta.

\section{- ALEGRIA}

Es una vivencia afectiva de placer y satisfacción que se asocia a una fuerza impulsora, de entrega y apertura hacia los demás y que puede percibirse en toda persona que la experimente a través de su apariencia, su lenguaje, en sus acciones constructivas y positivas, reflejando un estado de armonía y felicidad.

\section{- TRANQULIDAD Y CONFIANZA}

Son sentimientos positivos que reflejan un estado de bienestar, aceptación y seguridad, que se manifiesta cuando la persona vive momentos significativos y que le brinda un sentimiento de satisfacción, plenitud y equilibrio interior

\section{METODOLOGÍA}

Para la presente investigación se consideró una población de 198 estudiantes ingresantes de una institución educativa universitaria, que fueron evaluadas como parte de la evaluación del potencial de la ingresante EPI. Al realizarse el consentimiento informado con fines de investigación para el ESFA, se obtuvo una muestra de 85 estudiantes que representa el $45 \%$ de la población de ingresantes.
TABLA 1

Media y Desviación estándar de la edad

\begin{tabular}{lll}
\hline Edad & & \\
\hline $\mathrm{N}$ & Válidos & 85.00 \\
& Perdidos & 0 \\
Media & & 17.80 \\
Desv.tip. & & 1.75 \\
\hline
\end{tabular}

Se utilizó el instrumento Escala de Satisfacción Familiar por Adjetivos (ESFA) de J. Barraza y L. López (2010). La prueba está conformada por 27 items, cada uno formado por una pareja de adjetivos antónimos, correspondiendo la estructura a un diferencial semántico. La consistencia interna en la muestra de mujeres, informada por los autores se logró a través de la prueba Alfa de Cronbach (0.9771) y "Dos mitades" (0.9628). La estabilidad temporal se evaluó a través de la correlación test - retest $\left(0.7941^{*}\right)$. La validez de constructo se analizó a través del análisis factorial, apareciendo tres factores con un autovalor mayor de 1.00, siendo el I Factor el que explica por sí solo el $62.3 \%$. También se estimó validez de criterio con dos instrumentos que miden satisfacción familiar, obteniéndose $(r=0.7872)$ y $(r=0.6459)$. Finalmente se determinó validez discriminante con un pequeño grupo que se hallaba en terapia de orientación familiar.

Todos los cálculos estadísticos se realizaron por medio del paquete estadístico para las ciencias sociales SPSS, para Windows versión.

\section{Diseño de Investigación}

Se utilizó un diseño de tipo descriptivo, cuantitativo y cualitativo, porque se buscó evaluar y recolectar datos sobre el nivel de satisfacción familiar en una muestra de mujeres jóvenes y describir las dimensiones amor familiar y felicidad en esta muestra de estudio.

\section{RESULTADOS}

\section{NIVEL DE VALIDEZ Y CONFABILIDAD DE LA ESCALA DE SATISFACCIÓN FAMILIAR (ESFA)}

Se encontró que la escala posee validez de contenido adecuado, validado a través de la técnica de 
criterio de jueces. Y muy buena confiabilidad Alfa de Cronbach: 9:17 Guttman Split-Half de .934

\section{2.- GRADO DE SATISFACCIÓN FAMILIAR DE LA MUESTRA DE ESTUDIO}

En el grupo de estudio se halló un Alto nivel de Satisfacción Familiar, lo que refleja que los sentimientos despertados mientras se encuentran con su propia familia se orientan hacia los polos más favorables; existiendo agrado, apoyo, tranquilidad, bienestar, respeto y comprensión entre la familia.

TABLA 2

Medidas de Tendencia Central del Puntaje Total

\begin{tabular}{lc}
\hline & Puntaje Total \\
\hline Media & 138.79 \\
Desv.tip. & 15.37 \\
Mediana & 139.00 \\
Moda & 136.00 \\
\hline
\end{tabular}

3. COMPARACIÓN DE LA MUESTRA DE ESTUDIO CON LA MUESTRA ESPAÑOLA

En la comparación entre la muestra de estudio y la muestra española, hallamos que la media de la muestra de estudios, es más, siendo la desviación típica menor, debido a que los puntajes en su totalidad se hallan en una sola de las colas.

TABLA 3

Medidas de Tendencia Central de la muestra de estudio y la muestra española

\begin{tabular}{ccc}
\hline $\begin{array}{l}\text { Medidas de } \\
\text { Tendencia Central }\end{array}$ & $\begin{array}{l}\text { Muestra Peruana } \\
(\mathrm{N}=85)\end{array}$ & $\begin{array}{l}\text { Muestra Española } \\
(\mathrm{N}=148) \text { Mujeres }\end{array}$ \\
\hline Media & 138.79 & 122.26 \\
Desv.tip. & 15.37 & 26.30 \\
Mediana & 139.00 & 129.50 \\
Moda & 136.00 & 125.00 \\
\hline
\end{tabular}

4. TENDENCIAS DE LA SATISFACCIÓN FAMILIAR DEL GRUPO DE ESTUDIO DE ACUERDO A LAS CATEGORÍAS SEMÁNTICAS HALLADAS

Con respecto a la dimensión Amor Familiar está se halla a un nivel Alto, encontrándose que tanto el elemento de Holding como el de Sentido de pertenencia a la familia, se hallan en el nivel Alto.

En relación a la dimensión Felicidad se halla a un nivel Alto, encontrándose que tanto el elemento de Alegría como el de Tranquilidad, se hallan en el nivel Alto.

TABLA 4

Medidas de Tendencia Central de las dimensiones Amor Familiar y Felicidad

\begin{tabular}{lrrrrr}
\hline $\begin{array}{l}\text { Amor } \\
\text { Familiar }\end{array}$ & Holding & $\begin{array}{l}\text { Sent. De } \\
\text { Pert. }\end{array}$ & Felicidad & Alegría & $\begin{array}{l}\text { Tranq. y } \\
\text { Conf. }\end{array}$ \\
\hline 56.38 & 25.67 & 30.71 & 82.30 & 42.67 & 39.63 \\
3.47 & 3.66 & 4.32 & 8.48 & 4.38 & 4.98 \\
57.00 & 26.00 & 31.00 & 83.00 & 43.00 & 40.00 \\
64.00 & 30.00 & 33.00 & 81.00 & 48.00 & 40.00 \\
\hline
\end{tabular}

\section{CATEGORÍAS SEMANTICAS CONTENIDAS EN LOS ITEMS DE SATISFACCIÓN FAMILIAR POR ADJETIVOS}

Se realizó una búsqueda de los significados de cada uno de los reactivos, en relación al adjetivo positivo, realizándose una nueva agrupación.

a. Amor familiar, formado por dos grupos de significados:

i. Holding(ser acompañado, sostenido, protegido. 5 ítems: 2/5/8/21/24

ii. Sentido de pertenencia a la familia (animado, conocido, respetado, formando parte, estimulado) 6 ítems: 4/13/16/18/25/26

b. Felicidad, formado por dos grupos de significados:

i. Alegría, 8 ítems: 1/3/7/9/10/12/22/27

ii. Tranquilidad y confianza: 6/11/14/15/17/19/20/23 


\section{CONCLUSIONES}

1. La Escala de Satisfacción Familiar (ESFA) presenta una validez de contenido adecuada y una confiabilidad estadística global Alta.

2. En el grupo de estudio se halló un nivel Alto de Satisfacción Familiar

3. En la comparación entre la muestra de estudio y la muestra española, mostró en el grupo de estudio una media mayor y una desviación típico menor, debido a que los puntajes del grupo se agruparon en una sola de las colas.

4. El grupo de estudio presenta las dimensiones Amor Familiar y Felicidad, en un nivel Alto, revelando un Alto nivel de satisfacción familiar.

5. En el análisis cualitativo de la prueba ESFA, se determinaron las categorías semánticas Amor Familiar (formado por Holding y Sentido de pertenencia) y Felicidad (formado por Alegria y Tranquilidad y confianza).

\section{REFERENCIAS}

Alarcón Reynaldo (2000) Variables Psicológicas asociadas con la felicidad. Persona: 147-157. Lima

Alarcón, Reynaldo (2008) Psicología Positiva; un enfoque emergente. Revista Temática Psicológica 4. $\mathrm{N}^{\circ} 1,7-20$

APA (2010) Diccionario conciso de Psicología. México: Manual Moderno.

Arranz Freijo, Enrique; Oliva Delgado, Alfredo (2010) Desarrollo Psicológico de las nuevas estructuras familiares. Madrid: Pirámide.

Barraca Mairal, Jorge y López-Yarto Elizalde, Luis (2010) Escala de Satisfacción familiar por adjetivos. Madrid: Tea
Bermúdez C, Brik E, Terapia Familiar Sistémica Madrid: Síntesis

Céspedes Lorena, Roveglia Antonella y Cappari Norma (2014) Satisfacción familiar en adolescentes de un Colegio Privado de Asunción. Revista Centífica Eureka 11 (1): 37-51. Paraguay

De Zubiría Samper, Miguel (2007). Psicología de la felicidad. Colombia: Fundación Internacional de Pedagogía conceptual.

Diccionario de la Real Academia de la Lengua Española (2001) España

Dorsch, F (1997), Diccionario de Psicología. Barcelona: Herder

Guillén Rosario y colbs.(2011).Adaptación de la Escala de Satisfacción familiar por adjetivos (ESFA) en universitarios de Lima Metropolitana. Anales de Salud Mental/Volumen XXVII. Recuperado de: www.docenciaensaludmentalinsm.com/ojs 2.3.7/ index.php/docencia/article/view/36/31

Icart, A., Freixas, J. (2013) La Familia Comprensión Dinámica e Intervenciones Terapéuticas. Barcelona: Herder

La Fuente Benaches, María Josefa, Cantero López, María José (2010) Vinculaciones Afectivas. Apego, amistad y amor. Madrid: Pirámide.

Luna, A; Laca, F. \& Mejía, J. C. (2011). Bienestar subjetivo y satisfacción con la vida de familia en adolescentes mexicanos de bachillerato. Psicología Americana, 19(2), 17-26.

Organización Mundial de la Salud, Programa Envejecimiento y Ciclo Vital. Envejecimiento activo: un marco político. Rev Esp Geriatr Gerontol 2002; 37(S2):74-105.(*)

Sánchez, M. P. \& Quiroga, M. A. (1995). Relaciones entre satisfacción familiar y laboral: variables moduladoras. Anales de psicología, 11(1), 63-75. 